\title{
Eigenvalue Rigidity in Nonnegative Ricci Curvature
}

\author{
Mihriban Külahc1* Mehmet Bektaş \\ (Communicated by Yusuf YAYLI)
}

\begin{abstract}
Assume that $M$ is a compact orientable Lorentz manifold with timelike boundary $\partial_{t} M$. If the Ricci curvature of $M$ is bounded below by a positive constant $\mathbf{k}$, then $\lambda_{1}<(n-1) \max _{M}|H|-k$ where $\lambda_{1}$ is the first eigenvalue of the Laplacian of $M$.
\end{abstract}

Keywords: First Eigenvalue; Lorentz manifold; Nondegenerate timelike boundary.

AMS Subject Classification (2010): Primary: 53C24; Secondary: 53B30; 53C50.

\section{Introduction}

From the differential geometric point of view, the study of boundaries of Riemannian and Lorentz manifolds has its own interest. Many interesting results on Riemannian and Lorentz manifolds have been obtained by many mathematicians (see [2-5]).

In [4], Choi and Wang proved that if $M$ is a compact orientable hypersurface minimally embedded in $N$, then $\lambda_{1} \geq k / 2$ where $\lambda_{1}$ is the first eigenvalue of the Laplacian of $M$. Ho showed that if $M$ is a compact orientable hypersurface embedded in $N$, then $2 \lambda_{1}>k-(n-1) \max _{M}|H|$ in [5].

In this paper, we studied Lorentz manifold with timelike boundary $\partial_{t} M$ and we obtained rigidity theorem under the assumption on nonnegative Ricci curvature and we proved the rigidity theorem by considering Stokes theorem. The Stokes theorem is one of the most beautiful topics in mathematics. This beauty comes from bringing together a variety of topics: integration, differentiation, manifolds and boundaries. Furthermore, it is widely used in other sciences such as engineering and physics.

The main purpose of this paper is to carry out some results which were given in [4] and [5] to Lorentz manifold with timelike boundary $\partial_{t} M$.

\section{Preliminaries}

Let $R^{n}$ be the real n-dimensional vector space with standart basis $\left\{e_{1}, e_{2}, \ldots, e_{n}\right\}$. A inner product on $R^{n}$ is defined by

$$
\langle X, Y\rangle=\sum_{i=1}^{n} \varepsilon_{i} x_{i} y_{i}
$$

for each vectors $X=\left(x_{1}, x_{2}, \ldots, x_{n}\right)$ and $Y=\left(y_{1}, y_{2}, \ldots, y_{n}\right)$, where $\varepsilon_{1}=-1$ and $\varepsilon_{i}=1$ for $2 \leq i \leq n$ [3]. This inner product is called the Lorentz metric on $R^{n}$. Then the pairs $\left(R^{n},\langle\rangle,\right)$ is called the $\mathrm{n}$-dimensional Lorentz space and denoted by $L^{n}$. A vector $X$ in $L^{n}$ is respectively called spacelike, timelike or lightlike(null) if $\langle X, X\rangle>0$, $\langle X, X\rangle<0$ or $\langle X, X\rangle=0, X \neq 0[6]$.

Consider a Lorentz manifold $(M,\langle\rangle$,$) with boundary \partial M$. A normal vector to $\partial M$ at a point may have one of the three Lorentzian causal characters with respect to the induced metric on $\partial M$. Denote $\partial_{s} M, \partial_{t} M, \partial_{o} M$ the sets of the points where normal vectors are spacelike, timelike, lightlike(null) respectively. The subsets $\partial_{s} M$ and $\partial_{t} M$ are open in $\partial M$ and the subset $\partial_{o} M$ is closed in $\partial M$. Clearly, $\partial M$ consists of $\partial_{s} M, \partial_{t} M, \partial_{o} M$ and 
those subsets are pairwise disjoint. Consequently, $\partial M^{\prime}$ consists of $\partial_{s} M$ and $\partial_{t} M . \partial M^{\prime}$ is an open submanifold of $\partial M$ and may be considered as the nondegenerate boundary of $M$ and $\partial_{o} M$ is referred to as the degenerate boundary of $M$.

The main difficulty in stating a boundary for a Lorentz manifold is that the boundary may become degenerate at some of its points, and hence there exists no well-defined unit outward normal at such points.

Let $M$ be an n-dimensional Lorentz manifold with nondegenerate timelike boundary $\partial_{t} M$. Let $u$ be a function defined on $M$ smooth up to $\partial_{t} M$. Then $\Delta u$ and gradu denotes the Laplacian and the gradient of $u$ with respect to the Lorentz metric on $M$ respectively, similarly $L u$ and Gradu denotes the Laplacian and the gradient of $u$ with respect to the induced Lorentz metric on $\partial_{t} M$ respectively. For $x \in M$ and $X, Y \in T_{x} M$, we define the Hessian tensor $\operatorname{Hessu}(X, Y)=X(Y u)-\left(D_{X} Y\right) u$ where $D_{X} Y$ is the covariant derivative of the Lorentz connection of $M$. We denote the covariant derivative of the Lorentz connection of $\partial_{t} M$ by $\bar{D}_{X} Y$.

Let $\left\{e_{1}, e_{2}, \ldots, e_{n-1}, e_{n}\right\}$ be a local orthonormal frame such that at $x \in \partial_{t} M, e_{1}, e_{2}, \ldots, e_{n-1}$ are tangent to $\partial_{t} M$ and $e_{n}$ is the outward normal vector. We define the second fundemantal form $I I$ as, $I I(v, w)=\left\langle D_{v} e_{n}, w\right\rangle, v$ and $w$ are vectors tangent to $\partial_{t} M$, and $H$ to be the mean curvature, that is, $H=\frac{1}{n-1} \sum_{i=1}^{n-1} \varepsilon_{i} I I\left(e_{i}, e_{i}\right)$.

In differential geometry, the Stokes Theorem can be stated as follows [1]:

Let $M$ be a compact orientable k-dimensional manifold with boundary. If $w$ is $(k-1)$ - form on $M$, then

$$
\int_{M} d w=\int_{\partial M} w
$$

where $\partial M$ is the oriented boundary of $M$.

\section{Main Theorem}

Theorem 3.1. Suppose that $M$ is a compact orientable Lorentz manifold with nondegenerate timelike boundary $\partial_{t} M$. If the Ricci curvature of $M$ is bounded below by a positive constant $k$, then $\lambda_{1}<(n-1) \max _{M}|H|-k$ where $\lambda_{1}$ is the first eigenvalue of the Laplacian of $M$.

Proof. Let $f$ be the first eigenvalue of $M$, that is,

$$
\bar{\Delta} f+\lambda_{1} f=0 .
$$

Let $u$ be the solution of the Dirichlet problem such that:

$$
\left\{\begin{array}{l}
\Delta u=0, \text { in } M \\
u=f, \text { in } \partial_{t} M
\end{array}\right.
$$

Then $u$ is a function defined on $M$ smooth up to $\partial_{t} M$. Then

$$
\Delta u=\sum_{i=1}^{n} \operatorname{Hessu}\left(e_{i}, e_{i}\right)=\sum_{i=1}^{n+1} u_{i i},
$$

where $u_{i j}=\operatorname{Hessu}\left(e_{i}, e_{j}\right)$ for $i, j=1, \ldots, n$. When $x \in \partial_{t} M$, when $i \neq n$, we have $\operatorname{grad}\left(e_{i}, e_{i}\right)=\operatorname{Grad}\left(e_{i}, e_{i}\right)-$ $I I_{i i} e_{n}$, where $I I_{i j}=I I\left(e_{i}, e_{j}\right)$. Hence, by (3.2) and (3.3), when $x \in \partial_{t} M$,

$$
\begin{aligned}
\Delta u & =u_{n n}+\bar{\Delta} f+\sum_{i=1}^{n-1} I I_{i i} e_{n}(u) \\
& =u_{n n}+\bar{\Delta} f+(n-1) H u_{n},
\end{aligned}
$$

where $H$ is the mean curvature of $M$. Then, by (3.1) and (3.4), for $x \in \partial_{t} M$,

$$
u_{n n}=\lambda_{1} f-(n-1) H u_{n} \text {. }
$$


For $x \in M$, by the fact that $\Delta u=0$, we have $\frac{1}{2} \Delta|\operatorname{gradu}|^{2}=\sum_{i, j=1}^{n} u_{i j}^{2}+\sum_{i, j=1}^{n} R_{i j} u_{i} u_{j}$, where $R_{i j}=\operatorname{Ric}\left(e_{i}, e_{j}\right)$. By our assumption that Ricci curvature of $\partial_{t} M$ is bounded below by $k, \frac{1}{2} \Delta|\operatorname{gradu}|^{2} \geq \mid$ Hessu $\left.\right|^{2}+k \mid$ gradu $\left.\right|^{2}$, which implies

$$
\int_{M} \frac{1}{2} \Delta|\operatorname{gradu}|^{2} \geq \int_{M} \mid \text { Hessu }\left.\right|^{2}+k \int_{M}|\operatorname{gradu}|^{2} .
$$

When $i \neq n, u_{i n}=\operatorname{Hessu}\left(e_{i}, e_{n}\right)=e_{i}\left(e_{n} u\right)-\operatorname{grad}\left(e_{i}, e_{n}\right) u=e_{i}\left(u_{n}\right)-\sum_{j=1}^{n-1} I I_{i j} u_{j}$. On the other hand, using the Stokes theorem,

$$
\begin{aligned}
\int_{M} \frac{1}{2} \Delta|g r a d u|^{2} & =\int_{\partial_{t} M} \sum_{i=1}^{n-1} u_{i} u_{i n}+\int_{\partial_{t} M} u_{n} u_{n n} \\
& =\int_{\partial_{t} M} \text { Gradf.Gradun }-\int_{\partial_{t} M} \sum_{i, j=1}^{n-1} I I_{i j} u_{i} u_{j}+\int_{\partial_{t} M} u_{n} u_{n n} \\
& =\int_{\partial_{t} M} u_{n} \bar{\Delta} f-\int_{\partial_{t} M} I I(G r a d u, G r a d u)+\int_{\partial_{t} M} u_{n} u_{n n} \\
& =-\lambda_{1} \int_{\partial_{t} M} u_{n} f-\int_{\partial_{t} M} I I(G r a d u, G r a d u)-(n-1) \int_{\partial_{t} M} H u_{n}^{2} .
\end{aligned}
$$

Here we have used (3.1) and (3.5). By Stokes theorem and (3.2),

$$
\int_{M}|\operatorname{grad} u|^{2}=-\int_{M} u \Delta u+\int_{\partial_{t} M} u u_{n}=\int_{\partial_{t} M} u_{n} f .
$$

Hence, by (3.7) and (3.8), we have

$$
\begin{aligned}
\int_{M} \frac{1}{2} \Delta|\operatorname{gradu}|^{2}= & -\lambda_{1} \int_{M}|\operatorname{gradu}|^{2}-\int_{\partial_{t} M} I I(\text { Gradu,Gradu }) \\
& -(n-1) \int_{\partial_{t} M} H u_{n}^{2} .
\end{aligned}
$$

Combining (3.6) and (3.9), we obtain

$$
\begin{aligned}
\left(-\lambda_{1}-k\right) \int_{M}|g r a d u|^{2} \geq & \int_{\partial_{t} M} I I(\text { Gradu,Gradu })+(n-1) \int_{\partial_{t} M} H u_{n}^{2} \\
& +\int_{\partial_{t} M}|H e s s u|^{2} .
\end{aligned}
$$

Note that $\int_{\partial_{t} M} I I(G r a d u, G r a d u)=\int_{M} I I($ gradf, gradf $)$ and we can assume that $\int_{\partial_{t} M} I I(G r a d u, G r a d u) \geq 0$. On the other hand, by Stokes theorem and Holder's inequality

$$
\begin{aligned}
\int_{\partial_{t} M} H u_{n}^{2} & \geq-\max _{M}|H| \int_{\partial_{t} M} u_{n}^{2} \\
& =-\max _{M}|H|\left(\int_{M} u_{n} \Delta u+\int_{M} \text { gradun.gradu }\right) \\
& \geq-\max _{M}|H|\left(\int_{M}\left|g r a d u_{n}\right|^{2}\right)^{1 / 2} \cdot\left(\int_{M}|g r a d u|^{2}\right)^{1 / 2} .
\end{aligned}
$$


Here we have used (2). Now we consider two cases:

Case I: If $\int_{M}\left|g r a d u_{n}\right|^{2} \leq \int_{M}|g r a d u|^{2}$, then from (3.11), we have

$$
\int_{\partial_{t} M} H u_{n}^{2} \geq-\max _{M}|H| \int_{M}|\operatorname{gradu}|^{2} .
$$

Since $\int_{M}|H e s s u|^{2} \geq 0$ and $\int_{\partial_{t} M} I I(G r a d u, G r a d u) \geq 0$, by (3.10), we have

$$
\left(-\lambda_{1}-k\right) \int_{M}|g r a d u|^{2} \geq-(n-1) \max _{M}|H| \int_{M}|g r a d u|^{2} .
$$

Since $\int_{M}|\operatorname{gradu}|^{2}>0$, we get $\lambda_{1} \leq(n-1) \max _{M}|H|-k$.

Case II: If $\int_{M}\left|g r a d u_{n}\right|^{2} \geq \int_{M}|g r a d u|^{2}$, then from (3.11), we have

$$
\int_{\partial_{t} M} H u_{n}^{2} \geq-\max _{M}|H| \int_{M}\left|g r a d u_{n}\right|^{2} .
$$

Therefore, (3.10) implies that

$$
\begin{gathered}
\left(-\lambda_{1}-k\right) \int_{M}|g r a d u|^{2}+(n-1) \max _{M}|H| \int_{M}\left|g r a d u_{n}\right|^{2} \geq \\
\geq \int_{M} \mid \text { Hessu }\left.\right|^{2}+\int_{\partial_{t} M} I I(\text { Gradu, Gradu }) \geq 0 .
\end{gathered}
$$

Since $\int_{M}\left|\operatorname{grad} u_{n}\right|^{2} \geq \int_{M}|\operatorname{grad} u|^{2}$, we have

$$
\begin{gathered}
\left(-\lambda_{1}-k+(n-1) \max _{M}|H|\right) \int_{M}\left|g r a d u_{n}\right|^{2} \geq \\
\geq\left(-\lambda_{1}-k\right) \int_{M}|g r a d u|^{2}+(n-1) \max _{M}|H| \int_{M}\left|g r a d u_{n}\right|^{2} \geq 0 .
\end{gathered}
$$

Since $\int_{M}|g r a d u|^{2}>0, \int_{M}\left|g_{r a d} u_{n}\right|^{2} \geq \int_{M}|g r a d u|^{2}>0$ by our assumption. Hence, $\lambda_{1} \leq(n-1) \max _{M}|H|-k$.

Therefore, in both cases, we have $\lambda_{1} \leq(n-1) \max _{M}|H|-k$. We claim that it is impossible for the equality holds. Suppose not, then by above argument, we must have $\int_{M}|H e s s u|^{2}=0$. From this, we get $u_{i j}=0$ on $M$ for all $1 \leq i, j \leq n$. Since $u$ is smooth up to $\partial_{t} M$, we get $f_{i j}=0$ on $\partial_{t} M$ for $1 \leq i, j \leq n-1$, which implies that $L f=0$ which is impossible since $f$ is the first eigenfunction of $M$. This proves our claim. We have $\lambda_{1}<(n-1) \max _{M}|H|-k$ as required.

\section{Acknowledgments}

So long and thanks for all useful and constructive comments to referees.

\section{References}

[1] Abraham, R., Marsden, J.E. and Ratiu, T., Manifolds, Tensor Analysis and Applications, Second Press, Springer Verlag, New York, 1988. [2] Barros, A. and Bessa, G.P., Estimates of the first eigenvalue of minimal hypersurfaces of $S^{n+1}$, arXiv:math.DG/0410493v1, (2007). 
[3] Bektaş, M., The Reilly's integral formula on Lorentz manifolds with nondegenerate timelike boundary. Science and Engineering Journal of Firat University 10(2) (1998), 89-97.

[4] Choi, H.I. and Wang, A.N., A first eigenvalue estimate for minimal hypersurfaces. J. Differential Geom. 18(1983), 559-562.

[5] Ho, P.T., A first eigenvalue estimate for embedded hypersurfaces. Differential Geometry and its Applications (2007), doi:10.1016/j.difgeo.2007.11.019.

[6] O’Neill, B., Semi-Riemannian Geometry with Applications to Relativity, Academic Press, New York, 1983.

\section{Affiliations}

MIHRIBAN KÜLAHCI

AdDRESS: Firat University, Dept. of Mathematics, 23119, Elazığ-Türkiye.

E-MAIL: mihribankulahci@gmail.com

MEHMET BEKTAŞ

AdDRESS: Firat University, Dept. of Mathematics, 23119, Elazı̆̆-Türkiye.

E-MAIL: mbektas@firat.edu.tr 